\title{
Brasilia 50 años. Lo local y lo global
}

\author{
Brasilia 50 years. The local and the global
}

$<$ Resumen>

En el año 2007, conmemoramos el centenario de nacimiento de Óscar Niemeyer, y recordamos los cincuenta años del Concurso Público que eligió el «Plano Piloto» de Lucio Costa para orientar la construcción de Brasilia. Imaginada en el 1957, la «capital de la esperanza» llega a nuestros días con muchos problemas, pero también con muchas soluciones y/o aciertos.

$<$ Abstract $>$

2007, commemorated the centenary of the birth of Oscar Niemeyer, and we remember the fifty years of the Public Competition that chose the «Pilot Plan» by Lucio Costa to guide the construction of Brasilia. Conceived in 1957, the "capital of hope» arrives at the modern day with many problems, but also with many solutions and / or successes.

<PALABRAS CLAVE >

BRASIL / BRASILIA / CIUDADES PLANEADAS / LUCIO COSTA / ÓSCAR NIEMEYER

\section{-KEYWORDS >}

BRAZIL / BRASILIA / PLANNED CITIES /

IUCIO COSTA / OSCAR NIEMEYER

"Ni Chandigarh, la metrópolis que Nehru encomendó a Le Corbusier para sustituir la sede administrativa de Punjab; ni la nueva Bagdad, que Irak construye con recursos que hacen falta a la penuria de otras zonas de su territorio; ni las obras que Israel movilizó para improvisar en pocos años una Nación; o el esfuerzo de Japón para recuperarse, en lo urbano, de la decapitación atómica de sus ciudades; nada ha logrado la repercusión publicitaria tan destacada y sensacional en todos los centros del universo como el planeamiento y ejecución de Brasilia»**.

Osvaldo OrIco, 1961

\section{Una fiesta brasileña}

En 1957 el mundo conoció el proyecto vencedor del «Concurso Nacional do Plano Piloto da Nova Capital do Brasil». El anuncio (Edital) del concurso fue lanzado el 19 de septiembre de 1956' y la decisión fue divulgada oficialmente el 23 de marzo de $1957^{2}$. El jurado que escogió el proyecto de Brasilia fue establecido por la Companhia de Urbanização da Nova Capital (Novacap), con la relevante participación del arquitecto Óscar Niemeyer, a quien se le pidió la indicación de los jurados extranjeros.

\footnotetext{
Doctor en arquitectura (1993-1999), master en arquitectura (1990-1994) y graduado en arquitectura y urbanismo (1982-1987). Actual Director de la Facultad de Arquitectura y Urbanismo de la Universidad de Brasília (UNB). Profesor Adjunto del Departamento de Teoria e Historia en Arquitectura y Urbanismo de la Universidad de Brasília (UNB). Autor de inúmerables textos y articulos sobre arquitectura brasileña.

** Orico, 1961. p. 265

1 El anuncio (Edital) del Concurso fue publicado en el «Diario Oficial da União» el 30 de septiembre de 1956. Ver: Edital para o concurso nacional do plano piloto da nova capital do Brasil. Módulo, 8 de julio 1957.

2 Las dos Actas de la Comisión del jurado del Plan Piloto de Brasilia se publicaron en el «Diario Oficial da União» el 25 de marzo de 1957. Ver: Atas da comissão julgadora do plano piloto de Brasília. Módulo, 8 de julio 1957.
} 
Compusieron la comisión del jurado: el ingeniero Israel Pinheiro (Presidente de la Novacap y de la comisión, sin derecho a voto); dos representantes de la Novacap: los arquitectos Óscar Niemeyer y Stamo Papadaki (grecoamericano, autor de los primeros libros sobre la obra de Niemeyer ${ }^{3}$ ); dos representantes de entidades del rubro: el ingeniero Luiz Hidelbrando Horta Barbosa (del Club de Ingeniería) y el arquitecto Paulo Antunes Ribeiro (del Instituto de Arquitectos); y dos urbanistas extranjeros: William Holford (inglés, responsable por el Plan Regulador de Londres) y André Sive (francés, consejero del Ministerio de Reconstrucción de Francia). Es importante registrar que Niemeyer, en un primer momento, había indicado los nombres de Maxwell Fry (entonces Presidente de los CIAM) y Charles Asher (profesor de ciencias políticas en Nueva York) como representantes extranjeros.

Óscar Niemeyer era ya un arquitecto consagrado, cuando en el 56 fue invitado por el presidente de la República de Brasil para proyectar Brasilia y sus edificios.

«Óscar, esta vez vamos a construir la capital de Brasil, una capital moderna, la capital más bonita del mundo!» ${ }^{4}$, le dijo en la ocasión Juscelino Kubitschek de Oliveira (JK), Presidente brasileño. Niemeyer aceptó tratar solamente las cuestiones referidas a la arquitectura de la ciudad, rehusándose a la autoría del plan urbano de la futura capital.

Le Corbusier -quien tanto influiría en la arquitectura moderna de Brasil, y quien acababa de proyectar Chandigarh (1952)podría haber sido una opción, él mismo le había escrito a Juscelino, ofreciéndose para cumplir la tarea; pero no fue tomado en cuenta, sin embargo contaba con el apoyo del arquitecto Affonso Eduardo Reidy y del paisajista Roberto Burle Marx.

«Los tiempos eran otros, ni JK ni Niemeyer deseaban que una estrella extranjera intentara demostrar la pujanza de un Brasil moderno» ${ }^{5}$. En este sentido, cabe recordar los precedentes polémicos que envolvieron la autoría de los edificios del Ministério da Educação e Saúde (RJ, 1937-43) y de la sede de la Organización de las Naciones Unidas (NY, 1947) que probablemente incomodaron bastante a Óscar Niemeyer. Esta vez se trataba de un asunto correspondiente a Brasil, creada por y para los brasileños, con el fin de causar la admiración mundial.

\footnotetext{
4 Papadaki, 1950 y 1956.

5 Niemeyer, 1999; p. 109.
}

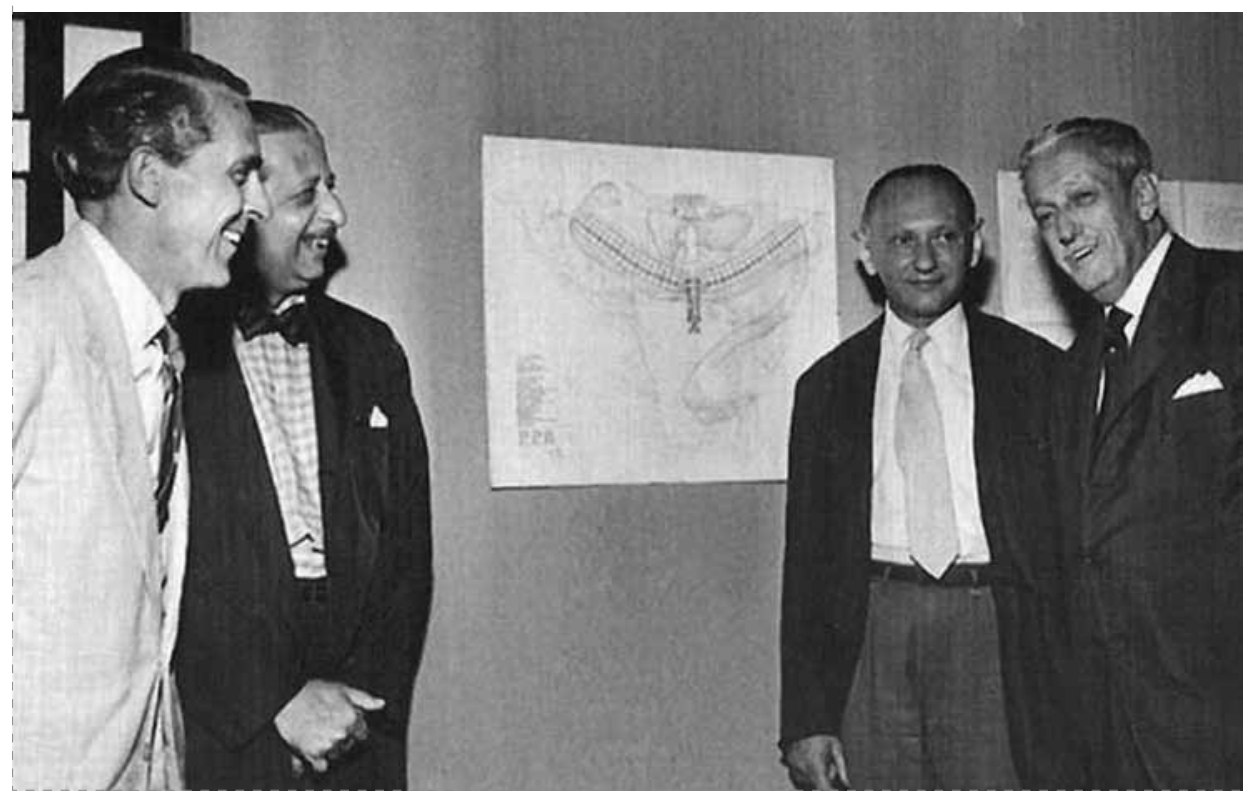

Brasilia Avenida W3 1957. Foto de Mário Montinell (Fuente: Archivo Público Federal)

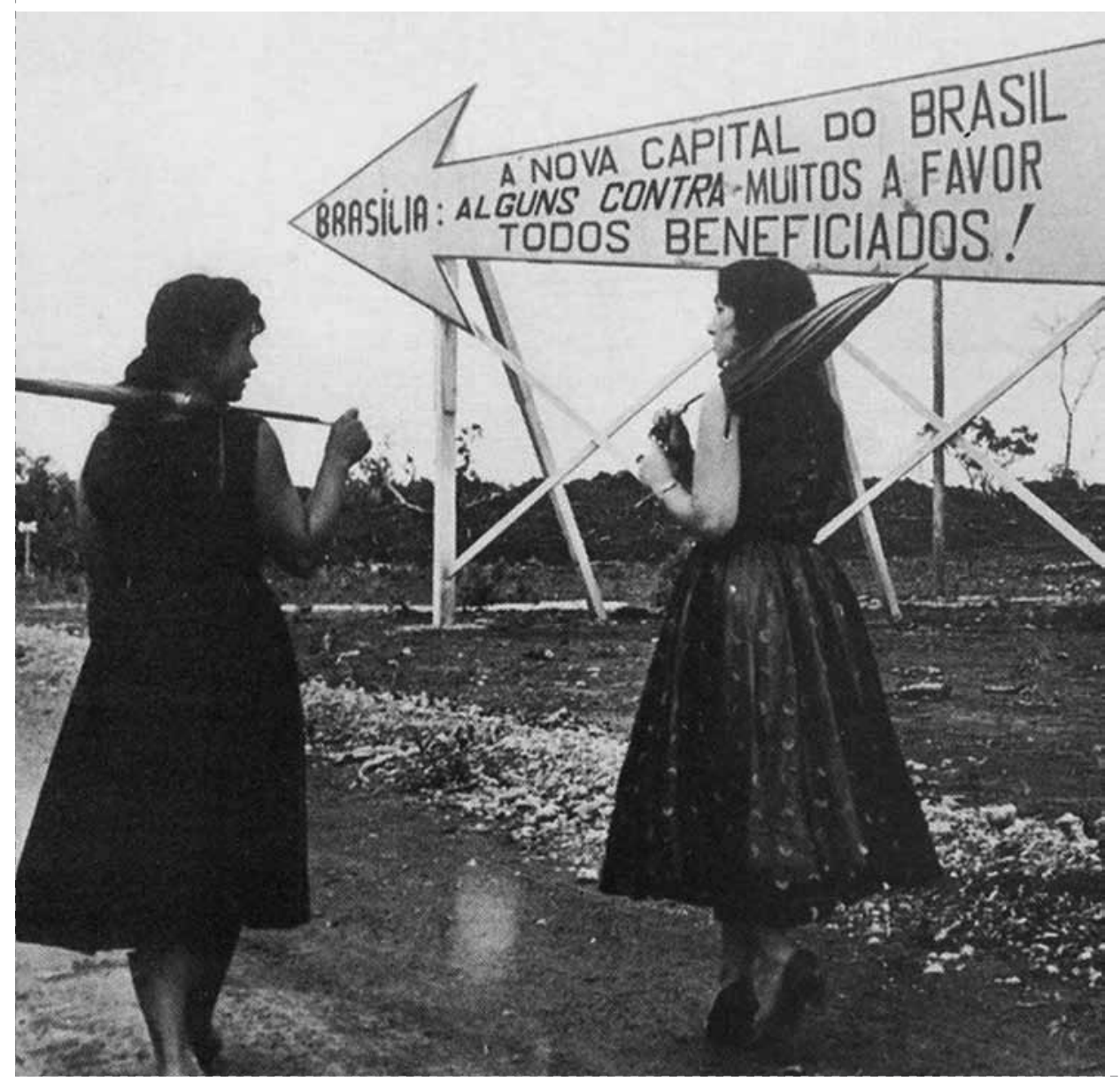


Brasilia, julio 1957, foto de Mário Montinelli (Fuente: Archivo Público Federal).

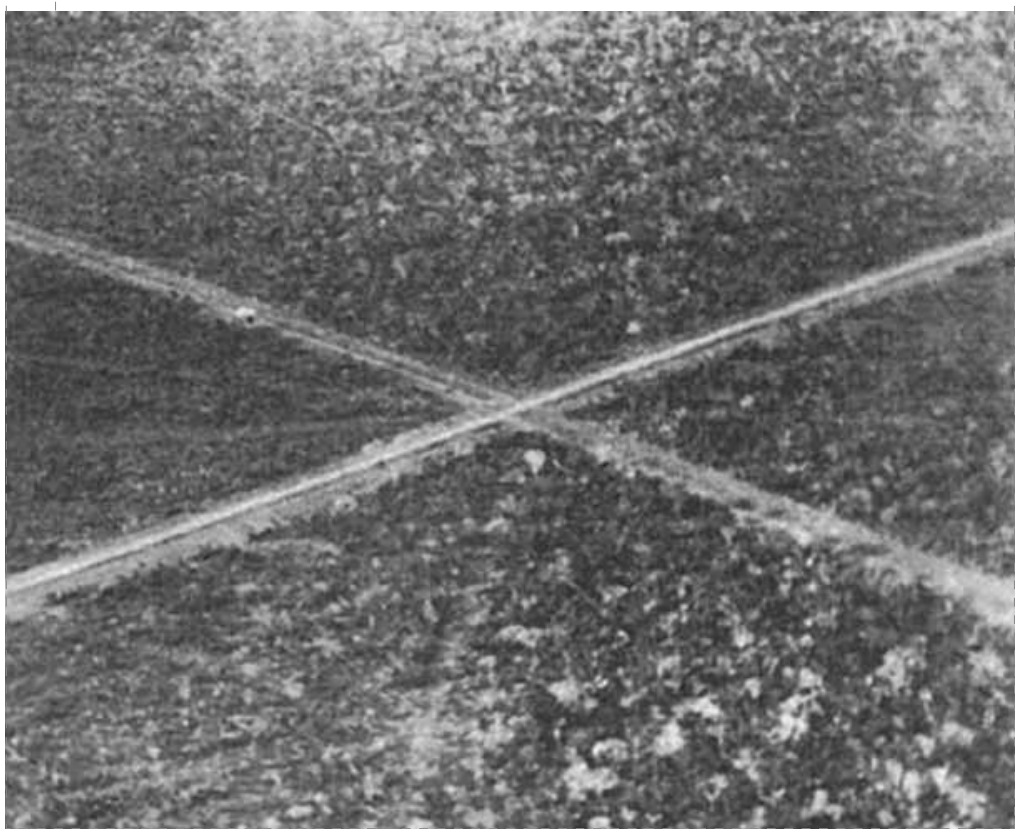

Belo Horizonte, 1987 (Fuente: Cediarte, FAU, UnB).

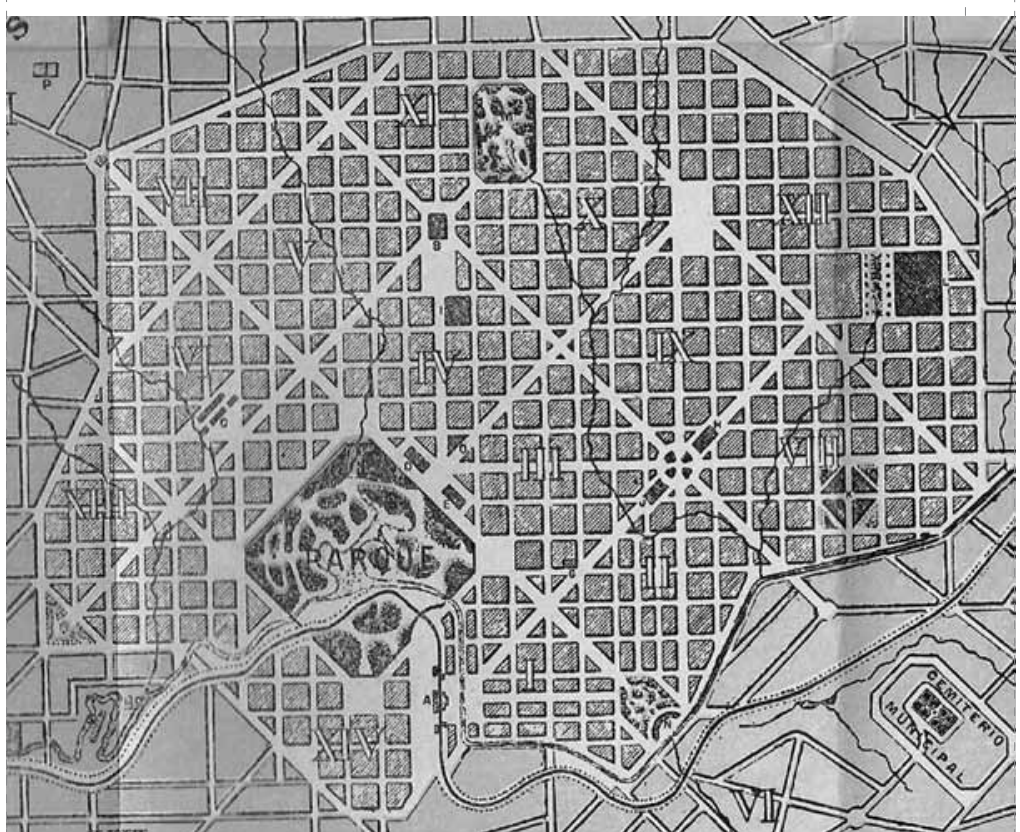

En palabras de JK, Brasilia representaría el esfuerzo conjunto de toda la Nación ${ }^{6}$. La publicación del anuncio (Edital) de 1956 definía que en el concurso para el Plano Piloto sólo podrían participar las «personas físicas y jurídicas domiciliadas en el país, que regularmente ejercían la ingeniería, la arquitectura y el urbanismo » ${ }^{7}$. Además, se exigía solamente el trazado básico de la ciudad y un relato justificativo.

Antes de la elección del Plano Piloto de la nueva capital de Brasil, Óscar Niemeyer ya había tratado de proyectar sus primeros monumentos. Nótese que ya había dibujado el Catetinho (la residencia provisoria del presidente de la República), el Palácio da Alvorada (su residencia definitiva) y el Brasília Palace Hotel (hospedaje para las visitas ilustres). Aún antes de la inauguración de la ciudad, ya había sido visitado y conocido el enorme espacio que ocuparía Brasilia, pudiéndose constatar el gigantesco esfuerzo que realizaba el país en la construcción de su capital.

Al Concurso Nacional do Plano Piloto da Nova Capital do Brasil se inscribieron sesenta y seis equipos, sólo veintiséis de los cuales terminaron por competir con sus respectivos proyectos, representados por innumerables profesionales de distintas formaciones. Los arquitectos más importantes del país también se avocaron a tan distinguida tarea: Rino Levi, los hermanos Roberto, Henrique Mindlin, Vilanova Artigas e Carlos Cascaldi encabezaron algunos de los equipos. Además se pueden considerar que los proyectos correspondían, sin excepción, a aplicaciones de los ideales del urbanismo funcionalista. En los distintos informes o relatos justificativos presentados, no hay una sola alusión o referencia a la construcción de una capital que correspondiera a una sociedad ideal, fundamentada en leyes justas y en instituciones político-económicas verdaderamente comprometidas con el bienestar de la colectividad, lo que permitiría hablar de una ciudad utópica, situación contraria de lo que representa Brasilia hoy en día. Hay tan solamente la expresión de una voluntad de hacer algo nuevo y mejor, premisa de todo y cualquier buen proyecto arquitectónico o de urbanismo.

Con respecto al anuncio (Edital), debo mencionar dos importantes omisiones: 1) no se hacía referencia a la población de la nueva capital; 2) no contemplaba una estructura administrativa estatal. Tales omisiones fueron sanadas por Óscar Niemeyer, quien en respuesta a consultas hechas, estableció en 500 mil el límite máximo de habitantes de la futura capital y una estructura administrativa equivalente a la entonces existente en Rio de Janeiro. Ante las consultas hechas a Niemeyer sobre cuestiones de división, partición y propiedad de la tierra, prefirió «aguardar a las sugerencias» ${ }^{8}$ de los competidores, es decir, todo podría ser propuesto.

Como se ha dicho, de los 66 inscritos en el concurso, 26 presentaron propuestas o planes y a su vez solo cuatro respondieron satisfactoriamente a los criterios establecidos por la comisión del jurado:

«...una Capital Federal -destinada a expresar la grandeza de una voluntad nacionaldeberá ser diferente a cualquiera ciudad de 500.000 habitantes. Además, la Capital, ciudad funcional, habrá de tener expresión arquitectónica propia. Su característica principal es la función gubernamental. A su alrededor se agruparán todas las otras funciones, y en ella convergerá todo... ${ }^{9}$.

La comisión eligió como vencedor al proyecto número 22, elaborado por Lucio Costa, que según se cree, no hubiese podido ser

Kubitschek, 1975; p. 5.

Edital para o concurso nacional do uplano piloto da nova capital do Brasil, Módulo 8 , julio 1957. p. 9.

8 Correspondencia al Presidente del IAB. Módulo 8, julio 1957; pp. 12 y 18.

Atas da comissão julgadora do Concurso do «Plano Piloto» de Brasília, Módulo 8, julio 1957; p. 18 
Gôiana, 1937 (Fuente: Cediarte, FAU, UnB).

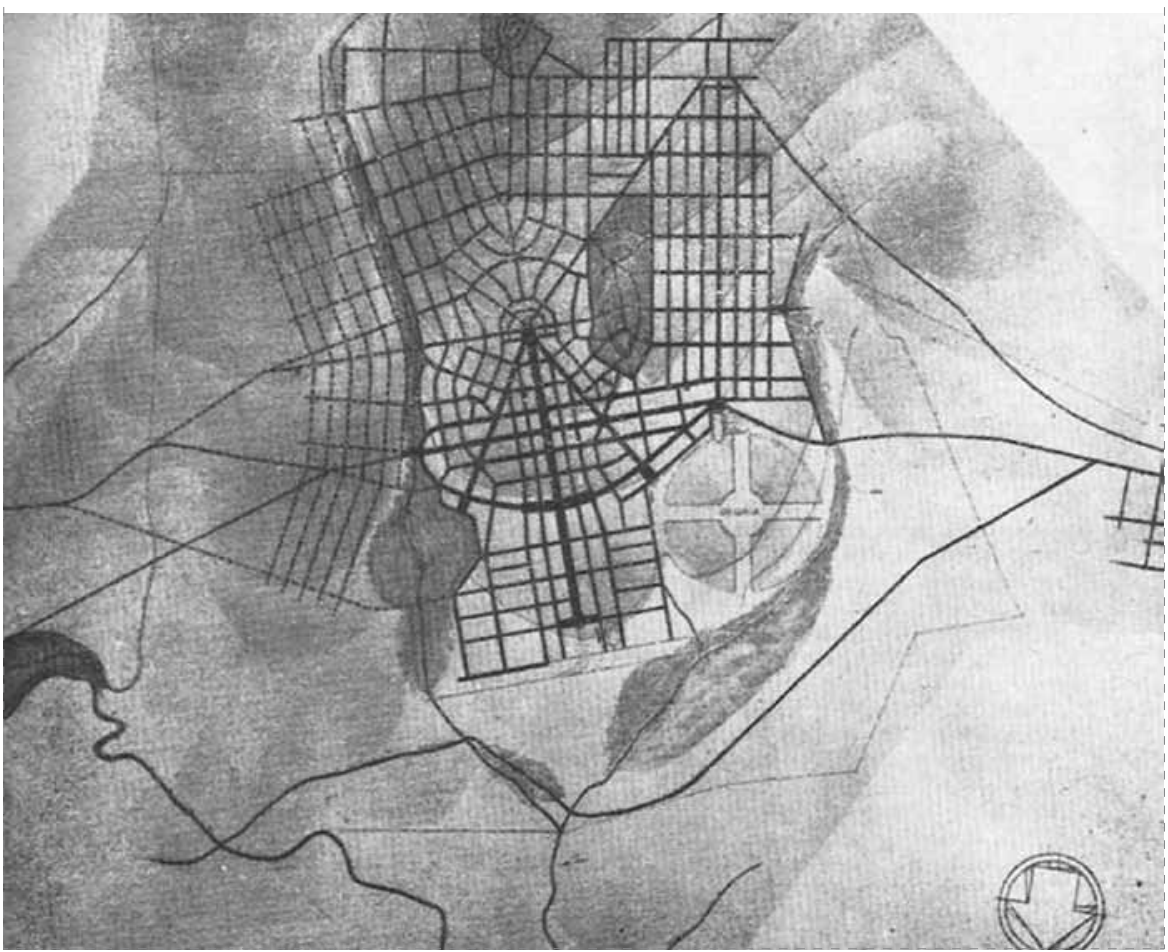

Palmas 5 Gôiana, 1990 (Fuente: Cediarte, FAU, UnB)

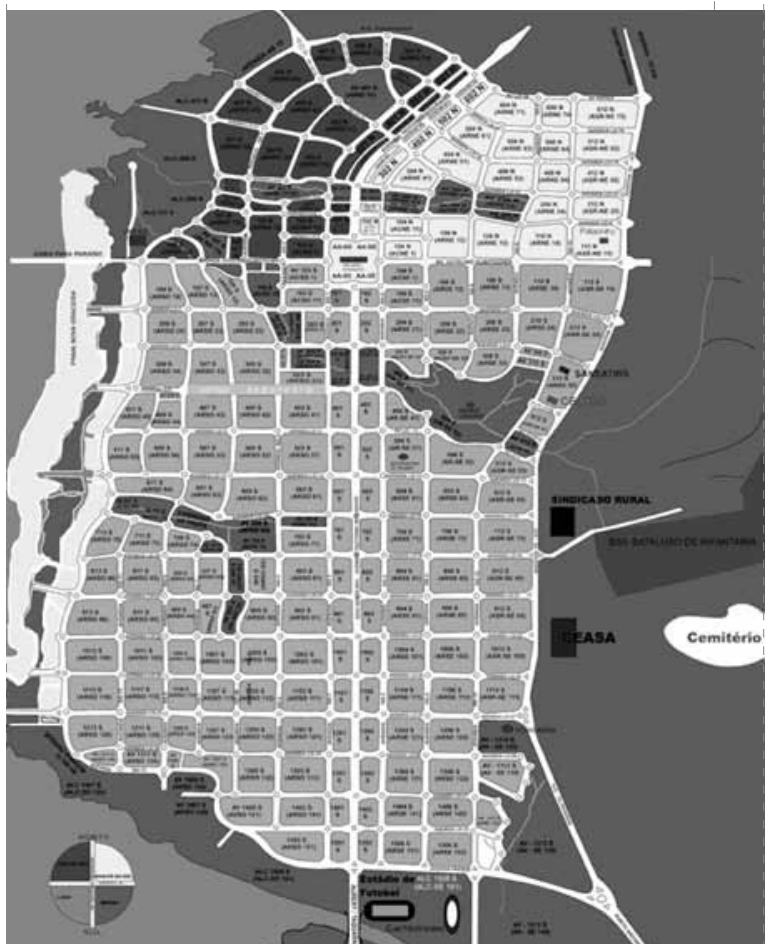

diferente pues ese proyecto era «el único a la altura para una capital administrativa de país». Aún más: «sus elementos pueden ser asimilados fácilmente: el plan es claro, directo y fundamentalmente simple, como por ejemplo, el de Pompéia, de Nancy, de Londres hecho por Wren, y el de París de Louis $X V{ }^{10}{ }^{10}$ Representaba un proyecto brasileño, lleno de características universales y se valía de todo un repertorio urbano significativo.

Hubo cierto constreñimiento causado por el voto contrario del representante del Instituto de los Arquitectos, Paulo Antunes Ribeiro, quien no había concordado con la metodología de elección de los vencedores, con respecto a eso Niemeyer se manifestó sin dejar de conmemorar el resultado:

«El resultado del concurso, a pesar de haber sido ganado honestamente, disgustó a algunos... Todavía me vienen a la memoria algunos incidentes, ciertos pasajes que casi lograron que dudara de muchas cosas... Pero, con el proyecto de Lucio Costa, todo se ha esclarecido. No sólo se trata de un admirable proyecto, sino también de un hombre puro y sensible, de un gran amigo con el que me podría entender» ${ }^{11}$.

La conmemoración no ocurrió de manera protocolar ni formal, así recuerda Niemeyer: "Fiesta de hombre es con una mujer!! ${ }^{12}$. Una fiesta mundana y brasileña, en un departamento en Rio de Janeiro, con mucha bebida, «media docena de conocidas», Niemeyer, Holford, Sivé y Papadaki... Y, como invitados especiales: el pintor Di Cavalcanti y el músico Ari Barroso. Mulatas y Aquarela do Brasil. «Una fiesta humana y cordial que sólo los surrealistas de París podrían haber concebido» ${ }^{13}$.

\section{Seis ciudades sobrepuestas}

Brasilia tiene que ser estudiada como un ejemplo más, o una consecuencia del gigantesco proceso de urbanización brasileño, caracterizado -entre otras cosas- por la concentración de población en los centros urbanos, por la creación y construcción de nuevas ciudades prácticamente de cero. Este proceso tuvo raíces históricas lejanas, pero tuvo un importante empuje a fines del siglo xIx y comienzos del siglo xx, con la construcción e inauguración de Belo Horizonte (1897); fue reforzado con la construcción e inauguración de Goiânia (1937); y alcanzó una etapa de divulgación internacional con la construcción e inauguración de Brasilia (1960). Proceso que sigue pujante en estos días, con la construcción e inauguración de otras ciudades, entre las que sobresale Palmas $(1990)^{14}$

10 Resumo das apreciações do júri do plano piloto de Brasília, Módulo 8, julio 1957; p. 13.

1 Niemeyer, 1961; pp. 12-13.

2 Niemeyer, 1999; p. 125.

13 Niemeyer, 1999; p. 126

14 Palmas estaba prevista en la Constitución de 1988. Fue proyectada por Luiz Fernando Cruvinel Teixeira y Walfredo Antunes de Oliveira Filho, en 1989, y «inaugurada» en mayo de 1990. Sobre la capital de Tocantins ver: Segawa, 1991. 
Belo Horizonte, la capital de Minas Gerais, fue al principio ${ }^{15}$ imaginada por el ingenieroagrimensor Aarão Reis desde 1893, e inaugurada cuatro años más tarde, en 1897. «El plan elaborado para Belo Horizonte resume una significativa parte de la cultura y de las preocupaciones estéticas del siglo XIX relativas a la ciudad ${ }^{16}$. En la capital minera es posible encontrar referencias a los principales experimentos urbanísticos realizados hasta entonces, como los planes para Washington (EUA) y La Plata (Argentina), los cuales le sirvieron de modelo.

Goiânia fue inicialmente ${ }^{17}$ imaginada por el arquitecto y urbanista Atílio Correia Lima, a partir de 1933, e inaugurada cuatro años después en 1937, justo cuando ocurrió la transferencia de la capital. Los autores del proyecto «dominaban los conocimientos teóricos y técnicos, indispensables para que se concibiera una ciudad moderna en aquel período. Ellos consideraron los factores topográficos, geológicos, hidrográficos, climáticos, históricos, culturales, sociales y económicos. Se atuvieron también a la división de una ciudad sectorizada por actividades (habitar, circular, trabajar y recrear); en la integración de los espacios urbanos y rurales; en el control de las tierras urbanas por el pode público; y en la legislación urbana» ${ }^{18}$. Es decir, Goiânia ha sido construida con sentimiento de realidad.

Antes de concebir Brasilia y en el mismo sitio de la futura Capital, habían sido proyectadas y no edificadas: la ciudad de "Planópolis», de autor desconocido (1791); la ciudad de «Brasilia», de Theodoro Figueiredo de Almeida (1929); la «Futura Capital Federal do Brasil», del urbanista Carmem Portinho (1938); y «Vera Cruz», del ingeniero José Oliveira Reis y los arquitectos Raul Penna Firme y Roberto Lacombe. A su vez, Brasilia fue imaginada en 1957 e inaugurada tres años después, en 1960. Como en los casos anteriormente citados, el proyecto también ha reflejado el pensamiento urbanístico entonces adoptado.
Sylvia Ficher y Pedro Palazzo, en el artículo Paradigmas urbanísticos de Brasília, identificaron algunas de las principales referencias, teóricas o prácticas, que pueden ser utilizadas para una mejor comprensión del Plano Piloto y para el entendimiento de su origen: la recuperación de formas de composición monumental; la especialización del sistema viario; el concepto de carreteras; las formas de ampliación de ciudades; la demarcación de zonas; y las distintas propuestas de tipos como ciudad linear, garden city, ville radieuse, new towns, entre otras. «Tal acervo era entendido como referencia y repertorio, aceptado en el plan teórico y legítimamente empleado en el dibujo de los espacios urbanos» ${ }^{19}$. En general gracias a lo anterior, es posible describir el Plano Piloto de Brasilia como la base de varias ciudades sobrepuestas ${ }^{20}$.

- La ciudad monumental, organizada a partir de dos ejes principales que se cruzan en distintos niveles y que definen una estructura viaria simétrica, en cuyo tejido se distinguen nítidamente los espacios de representación del poder, tanto nacional como local;

- La ciudad de carreteras o viaria estructurada por una trama regular y jerarquizada a través de vías especializadas de circulación;

- La ciudad funcional, con destinaciones específicas para sus diferentes partes, las cuales reciben tratamientos claramente diferenciados:

- La ciudad parque, caracterizada por grandes áreas verdes y cuyas edificaciones no obedecen al régimen de división tradicional en lotes individuales;

- La ciudad radial, organizada en grandes manzanas, verdaderas unités de voisinage, en que impera la separación entre peatones y coches;

- La ciudad central, para ampliarse mediante el aumento de nuevos núcleos distintos, las ciudades-satélites.

\section{El contrapunto local}

La lectura atenta del relato del Plano Piloto de Brasilia, de Lucio Costa, permite observar que cuando propone el nuevo plan, enumera los tipos adoptados (una ciudad en cruz, por ejemplo) y hace referencia a algunos modelos urbanos que le gustaría ver reproducidos en su ciudad: la mezcla en términos adecuados de «Piccadilly Circus», "Times Square» y «Champs Elysées», las «travesías en el género tradicional de la calle del Ouvidor», los «callejones venecianos», las «loggias» italianas, los terraplenes de los palacios orientales, las ideas fundamentales de la técnica de las carreteras norteamericanas, los enormes céspedes ingleses, entre otros. Son tomados como ejemplos que denotan la tentativa de reproducir soluciones espaciales definidas y datadas socialmente.

Al inicio de su relato justificativo, Lucio Costa recordó que José Bonifácio en 1823, ya había propuesto la transferencia de la Capital brasileña para el centro del país (Goiás) y había sugerido también la denominación de Brasilia. En la presentación de la solución adoptada en su plan, explicó que la ciudad «nacía del gesto primario de quien señala un lugar o de quien lo toma como preferencia: dos ejes cruzados en ángulo recto, como si fuera la propia señal de una cruz». De esta forma, el autor del «Plano Piloto» definió su campo de relación con la historia y con la tradición ${ }^{21}$. En otro momento, en el pequeño texto titulado Ingredientes da concepção urbanística de Brasília ${ }^{22}$, Lucio Costa que afirma que la capital es una creación original, nativa y brasileña, indicando que la ciudad de Diamantina, en Minas Gerais es una de sus fuentes de inspiración. Podemos entender que la pureza de la ciudad minera no fue citada de manera aleatoria: ante los ejes y las perspectivas de París, los grandes céspedes ingleses, los terraplenes y los arrimos chinos, las carreteras y los viaductos norteamericanos, Diamantina es el contrapunto local, es la marca de la tradición. En el texto, Costa no citó las probables influencias de teorías de urbanismo europeo y omitió intencionalmente a Le Corbusier y los postulados del urbanismo de los Congresos Internacionales de Arquitectura

\footnotetext{
15 En 1895, Aarão Reis fue reemplazado por Francisco Bicalho.

16 Leme, 1999; p. 222

17 En 1936, Atílio Correia Lima fue reemplazado por Armando Augusto Godoy.

18 Leme, 1999; p. 226

9 Ficher, Palazzo, 2005.

Schlee, Ficher, 2006.

Schlee, 2003.

22 Costa, 1995
} 


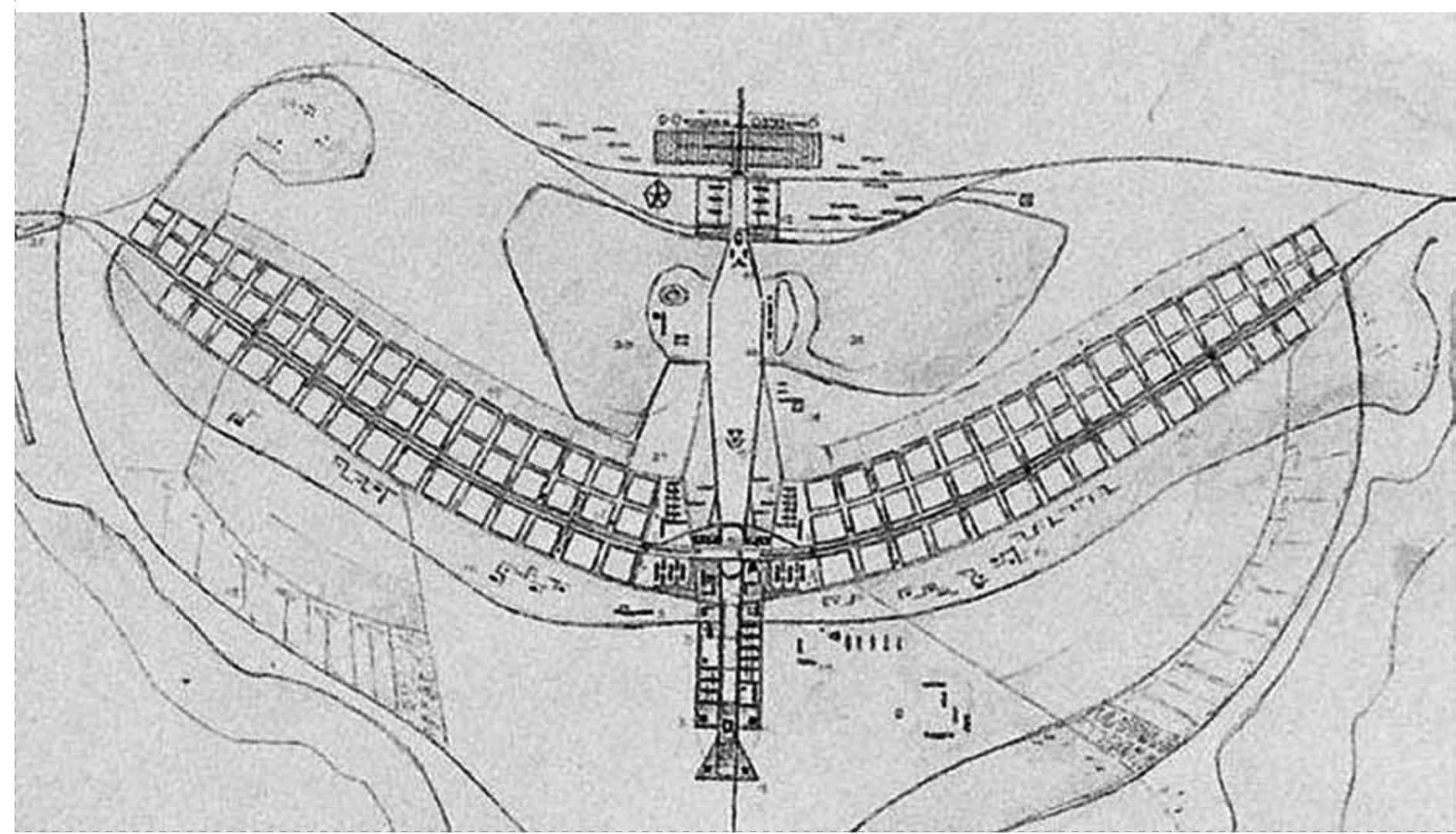

Moderna (CIAMs). Haciéndolo, no sólo refuerza la idea de la originalidad nativa (el local ante el global), sino también la del genio nacional, es capaz de -a partir de simples experiencias perceptivas ${ }^{23}$ y de determinadas condiciones locales de carácter material, social y temporal, producir las respuestas más creativas y sorprendentes. Brasilia, como ha definido su autor, es una creación «original y nativa». Original, en el sentido de lo que es hecho por primera vez, de lo que tiene carácter propio, de lo que busca no imitar o seguir a nadie y de lo que puede servir de modelo. Nativa, en el sentido de lo que es natural, de lo que no es extranjero, de lo que es sencillo y no artificioso, y que pertenece a una tierra o a un país. Entonces de la misma forma que tenemos Aleijadinho y Niemeyer, inventamos a Diamantina y a Brasilia.
En 1922, a partir de una indicación del profesor y amigo José Mariano Filho, Lucio Costa viajó por primera vez a Minas Gerais. Patrocinado por la Sociedade Brasileira de Belas Artes y en sintonía con el movimiento que plegaba el rescate de la arquitectura colonial (o neocolonial), fue a estudiar en terreno las distintas manifestaciones de la arquitectura minera. Aunque haya visitado las principales ciudades históricas mineras, eligió Diamantina, tierra natal de Juscelino Kubitschek y donde, durante la década del 50, Óscar Niemeyer habría de construir una escuela, un club y un hotel ${ }^{24}$. Lucio Costa afirmó con respecto a Diamantina:

«...llegando allá, me dí una zambullida en el pasado, en el sentido más despojado, más puro; un pasado de verdad, que yo ignoraba, un pasado que era nuevo y irrevelado para mí. Ha sido como una revelación: casas, iglesias, posadas de troperos... Y mal sabía yo que, 30 años después, iba a proyectar nuestra capital para un muchacho de mi edad nacido allí» ${ }^{25}$.

\section{La conexión establecida con Juscelino} Kubitschek es significativa, pues ha sido mediante la voluntad e influencia política de JK, que Niemeyer pudo construir sus principales obras en Pampulha, revelándose ante el mundo. Por otro lado, fue el presidente quien tornó posible la construcción de la capital proyectada, de manera que Diamantina es el contrapunto local, especialmente gestado por Lucio Costa.

\footnotetext{
23 Lucio Costa habla de sus recuerdos de París, de los inmensos céspedes ingleses de su infancia, de la pureza de Diamantina de los años veinte, de fotografías de China de principios del siglo xx, y de un viaje que hizo con sus hijas a Estados Unidos de América.

24 La escuela Júlia Kubitschek, el Club Diamantino y el Hotel Tijuco.

25 Costa, 1995.
} 


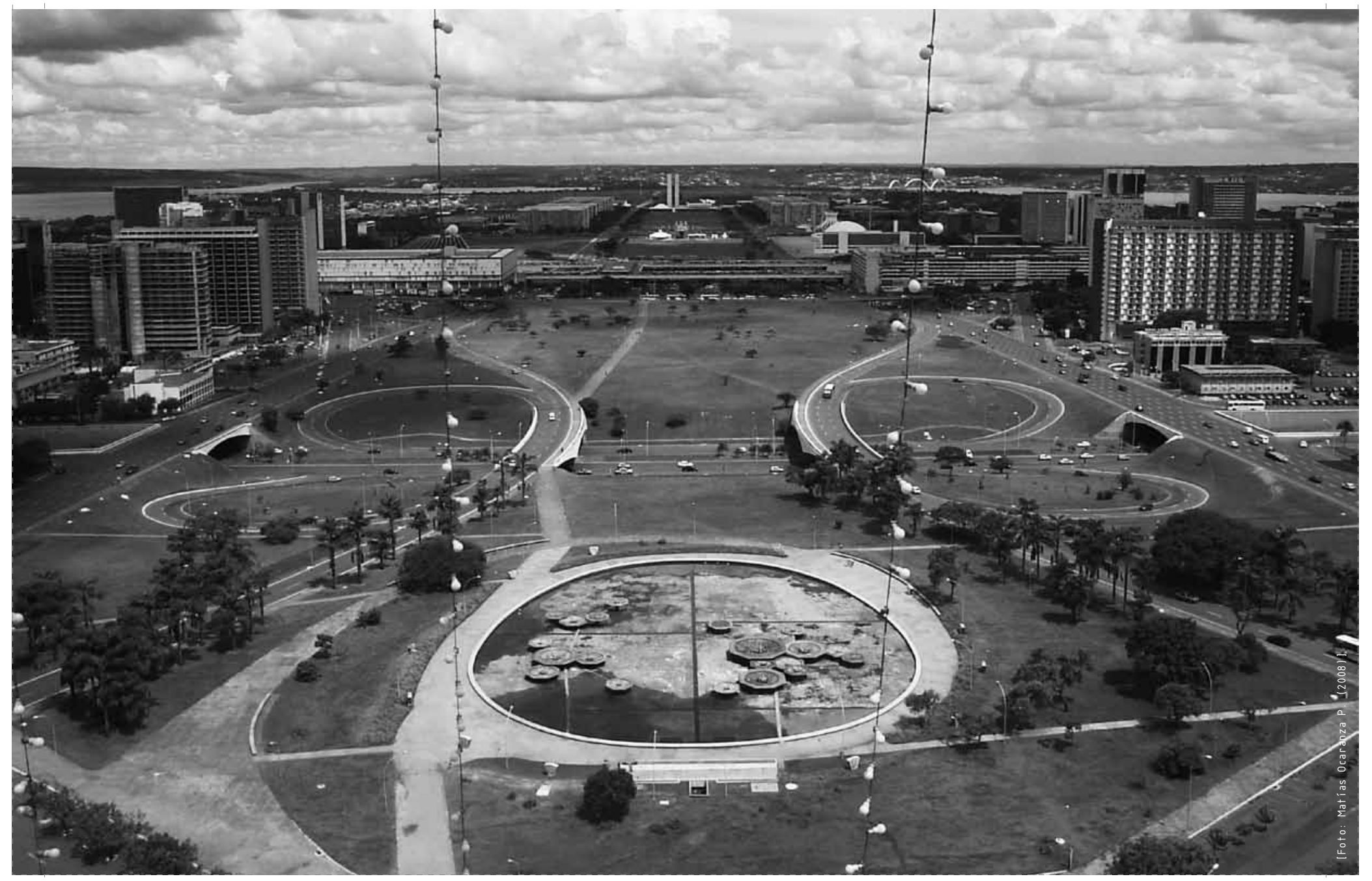

Congreso Internacional Extraordinario de Críticos de Arte

Simultáneamente a la construcción e instalación de la nueva Capital, el gobierno brasileño desarrolló un intenso y adecuado programa de divulgación y propaganda de Brasilia. Además de publicaciones oficiales, como la revista Brasília (publicación mensual de la Novacap) o el libro Brasil, capital Brasília, de Osvaldo Orico, se hicieron varias exposiciones que circularon por capitales de Europa y América Latina, de manera que poco a poco, la comunidad internacional y nacional fue tomando conocimiento de lo que se hacía en el Planalto Central de Brasil. Aunque la acción que causó más impacto internacional, fue la realización, entre el 17 y el 25 de septiembre de 1959, siete meses antes de la inauguración de la Capital, el Congreso Internacional Extraordinario de Críticos de Arte.

El acontecimiento tuvo por sede Brasilia, São Paulo y Rio de Janeiro, permitiendo que muchos de los participantes conocieran, en terreno, Brasilia. El principal tema llevado a discusión - «Brasília, cidade-síntese das artes»- fue propuesto por el organizador del Congreso, el crítico brasileño Mário Pedrosa, y sirvió de pretexto de visita de los congresistas a Brasilia. La lista de los participantes es sorprendente, tanto por la cantidad como por la calidad de los expositores: Alberto Sartoris, Amâncio Williams, André Bloc, André Chastel, Bruno Zevi, Carola Giedion (arquitecta y esposa de historiador), Charlotte Perriand, Eero y Aline Saarinen, Françoise Choay, Gillo Dorfles, Giulio Carlo Argan, Jean Prouvé, Meyer Shapiro, Stamo Papadaki, Tomás Maldonado y William Holdorf, entre otros ${ }^{26}$. Por su parte Brasil estuvo representado por Lúcio Costa, Óscar Niemeyer, Mário Barata, Ítalo e Quirino Campofiorito, Carlos Cavalcanti, Ferreira Gullar, Joaquim Cardoso, José Roberto Teixeira Leite, Fayga Ostrower y Dom Clemente Maria da Silva-Nigra, entre otros.

26 A presente listagem foi montada a partir de um documento original intitulado Relação de Membros do Congresso Internacional Extraordinário de Críticos de Arte. AICA, DOC.27-P. Osvaldo Orico acrescenta à listagem, os importantes nomes de Alvar Aalto, Richard Neutra e Sigfried Giedion. 


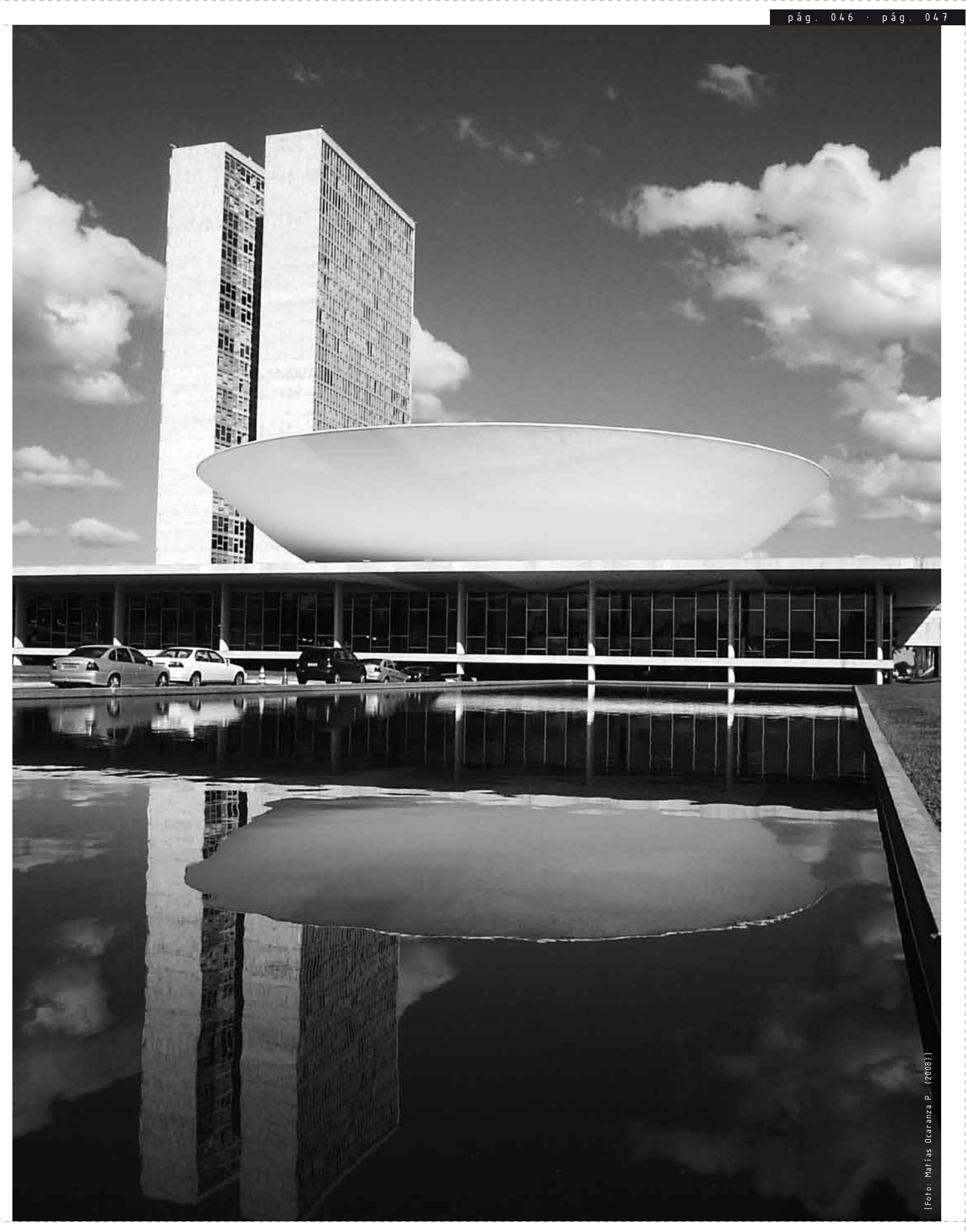


El encuentro de la intelectualidad artística con Brasilia fue impactante. Mário Pedrosa recuerda que «la mayoría de los delegados extranjeros traía en mano muchas críticas, sin embargo tuvo que revisarlas o cambiarlas, en la contradictoria presencia con la realidad ${ }^{27}$ Entre los pronunciamientos entonces registrados (casi en su totalidad elogios), se destacó el de Bruno Zevi. El autor de Saber ver la arquitectura, inició su discurso preguntándole al público quien criticaría: «A Dr. Lucio Costa o a Óscar Niemeyer?» Para sentenciar de inmediato:

«...los defectos de Brasilia son los defectos de nuestra cultura. Somos responsables. Si hay algunos defectos, resultan del hecho de que Brasilia reproduce físicamente los problemas que no sabemos resolver, que no resolvimos -ninguno de nosotros- en cualquier parte del mundo ${ }^{28}$.

Bruno Zevi habló de crisis, de crisis cultural, crisis de la urbanística y de la arquitectura. Enfático, llegó a recordar a Philip Johnson «la arquitectura moderna se acabó, y el Seagram Building es el último ejemplo de eso» ${ }^{29}$. Su inquietud tenía fundamento, o bien muchos fundamentos. Brasilia fue imaginada, dibujada y construida en un momento crucial de la historia de la arquitectura. Al mismo tiempo que se comenzaba a construir el mayor monumento del modernismo funcionalista, una significativa parte de las teorías que sustentaban al proyecto, pasaban a ser cuestionadas. En 1954 Philip Johnson había proferido la palestra titulada The seven crutches of modern architecture, en la formalista Universidad de Yale; en 1955 y 56, James Stirling (re)analizaría críticamente la obra de Le Corbusier ${ }^{30}$. en1960, Kevin Lynch publicó The image of the city; en 1961, Jane Jacobs, lanzó The death and life of great american cities; en 1962 Aldo van Eyck publicó el artículo sobre el Team $10^{31}$. En la década de 60, publicaron sus trabajos Christopher Alexander, Christian NorbergSchulz, Aldo Rosi, Robert Venturi y Charles Jencks, entre otros, considerados por sus publicaciones «clásicas». Las preocupaciones de tales autores, por cierto, no estaban en la pauta de Lucio Costa, y aún hoy no habrán de estarlo con la obra de Óscar Niemeyer.

Cuando estaban desenvolviendo el Plano Piloto de Brasilia, Lucio Costa estaba empeñado en resolver un enorme problema: como proyectar una capital para su país. No una ciudad moderna cualquiera, no solamente una URBS, sino una CIVITAS.

Lucio Costa proyectó la capital de Brasil y no la capital de la Esperanza. «Brasilia, la capital de Brasil, ha sido planeada y construida entre 1956 y 1960 como la materialización de los ideales de la ciudad modernista. Si Brasilia hubiera permanecido como un sueño, pasaría como un simple incidente local sin mayor interés, pero Brasilia no es un sueño y sin duda no es una pesadilla. Es una ciudad viva, en constante crecimiento y transformación, luchando entre un esquema fijo de modernidad y las necesidades de cada día »32. Tenemos que encararla de frente para estudiarla y conocerla mejor, hacernos de coraje para proponer y establece las modificaciones y transformaciones que se hagan necesarias, como también saber aprovechar mejor sus calidades incuestionables y extenderlas a un siempre creciente número de personas.

En la apertura del Congreso Internacional Extraordinario de Críticos de Arte, Lucio Costa dejó el siguiente mensaje para sus colegas:

"Hay que discutir y discordar con ganas. Ustedes son críticos; la insatisfacción es vuestro clima, pero de una cosa estoy seguro y es la presencia de Ustedes frente a Brasilia como testigo, comprueben lo que viene ocurriendo en muchos sectores de nuestras actividades, nosotros ya no exportamos sólo café, azúcar y cacao sino que también dimos de comer un poco a la cultura universal»33.

\section{Referencias bibliográficas}

Lauro Cavalcanti. Brasília: A construção de um exemplo. Arcos. Volume I, número único, 1988
Lucio Costa. Registro de uma Vivência. São Paulo: Empresa das Artes, 1995.

Sylvia Ficher, Pedro Paulo Palazzo. Os paradigmas urbanísticos de Brasília. Cadernos PPG-AU / FAUFBA. Edição Especial: Urbanismo Modernista 1930-1960, 2005

Antônio Houaiss, Mauro de Salles Villar. Dicionário Houaiss da língua portuguesa. Rio de Janeiro: Objetiva, 2001.

Juscelino Kubitschek. Por que construí Brasília. Rio de janeiro: Bloch, 1975; p. 5.

Maria Cristina da Silva Leme. Urbanismo no Brasi 1895-1965. São Paulo: FUPAM/Nobel, 1999.

Óscar Niemeyer. As curvas do tempo. Memórias. Rio de Janeiro: Revan, 1999.

Óscar Niemeyer. Minha experiência em Brasília. Rio de Janeiro: Vitória, 1961

Ósvaldo Orico. Brasil, capital Brasília. Rio de Janeiro: Record, 1961.

Stamo Papadaki. Óscar Niemeyer: Works in progress. New York: Reinhold, 1956.

Stamo Papadaki. The work of Óscar Niemeyer. New York: Reinhold, 1950.

Mário Pedrosa. Dos murais de Portinari aos espaços de Brasília. São Paulo: Perspectiva, 1981.

Andrey Schlee, Sylvia Ficher. Vera Cruz, futura capital do Brasil, 1955, Inédito, 2006.

Andrey Schlee. Lucio Costa, o senhor da memória. JORNAU 2003; 6

Hugo Segawa. Palmas, cidade nova ou apenas uma nova cidade? Projeto 1991; 146

James Stirling. From Garches to Jaoul: Le Corbusier as domestic architect in 1927 and 1953. The Architectural Review 1955; 705.

James Stirling. Ronchamp: Le Corbusier's Chapel and the crisis of rationalism, The Architectural Review 1956; 711.

Aldo Van Eyck. Team 10 Primer. Architectura Design, 1962.

Ruth Verde Zein. O lugar da crítica. Porto Alegre: Ritter dos Reis, 2001; p. 172.

Bruno Zevi. A dimensão das estruturas urbanísticas. Trabalho apresentado durante - Congresso Internacional Extraordinário de Críticos de Arte. AICA, DOC.22-P, 1959.

27 Pedrosa, 1981; p. 366

28 Zevi, Bruno citado por Pedrosa, 1981; p. 370.

29 Zevi, 1959

30 Stirling, 1955 y 1956.

31 Van Eyck, 1962

32 Zein, 2001; p. 172.

з3 Costa, 1995. 